\title{
Review of: "Lack of evidence for a role of anthrax toxin receptors as surface receptors for collagen VI and for its cleaved off C5 domain (endotrophin)"
}

\author{
James Bann ${ }^{1}$ \\ 1 Wichita State University
}

Potential competing interests: The author(s) declared that no potential competing interests exist.

The study by Przyklenk et al., provide experimental evidence that suggests that TEM8 or ANTXR1 (tumor endothelial marker 8/Anthrax toxin receptor 1 ) does not bind to the C5 domain of collagen type VI, also known as endotrophin. The C5 domain has been shown to encode a 76 amino acid, disulfide bonded protein that is cleaved off of the a3 chain of type VI collagen during microfibril assembly (Mayer et al., Eur J. Biochem. 1994; Lamande, S. J Biol Chem 2006). Previous studies by Nanda and co-workers in the St Croix lab provided evidence using a yeast two-hybrid screen that the ANTXR1 von Willebrand factor A domain (which contains the MIDAS site and allows for interaction with anthrax toxin protective antigen (PA)) bound to the C5 domain of collagen type VI (Nanda et al., Cancer Res. 2004). Binding was shown to be dependent on an intact C5 domain, as the product of the 205 bp cDNA from yeast two-hybrid screen, but not a 60 bp shortened cDNA product, could upon expression in HEK 293 cells co-precipitate ANTXR1. Furthermore, in situ hybridization demonstrated that ANTXR1 and type VI collagen were seemingly colocalized in intact tissues, suggesting that the two interacting partners may be coordinately regulated. In the present study, however, the authors are unable to detect binding between the C5 domain and ANTXR1. The positive control for binding to ANTXR1 in these studies is PA, which binds ANTXR1 and ANTXR2 with high affinity. However, ANTXR1 and ANTXR2, which share homology to integrins, are likely to bind collagen with much lower affinity. As an alternative positive control for binding the C5 domain the authors also utilize NG2, which has been shown in previous studies to bind type VI collagen as well as other collagens (II and V, for instance) (Burg et al., J. Biol Chem 1996). However, in the cell binding or immunoprecipitation studies in this manuscript, the isolated C5 domain does not bind NG2. In fact, the only case where collagen VI binds to NG2 is in the tetrameric microfibril. In the study by Burg et al., only the processed type VI was shown bind to NG2, not the microfibril, so it is perhaps not surprising that NG2 does not bind C5. So, in fact there is no control for an intact C5 in this study. There were no studies for instance to show that antibodies directed against the C5 domain also cross-react with the collagen VI microfibrils, and those antibodies could not cross-react with processed type VI collagen (in the same manner as was done by Mayer et al., Eur J. Biochem. 1994). It would be helpful if the current authors could show that the recombinant C5 (also produced in HEK 293 cells) was disulfide bonded by comparing nonreduced and reduced migration in an SDS-PAGE gel. 
In Figure 3D the authors attempt to evaluate an interaction between ANTXR1 or ANTXR2 and C5 using a pull- down assay. For this assay cell lysates from HEK293T cells or HEK293T cells expressing HIS-tagged ANTXR1 or ANTXR2 cells were mixed with Ni-NTA beads as well as collagen VI and subsequently protective antigen. After pulling down the ANTXR1 proteins, the authors note only PA can co-precipitate, but C5 and alpha 1 collagen 6 cannot. However, in this experimental setup, because collagen is likely to bind the ANTXR proteins at the same region of the VWA domain as PA (both depend on binding to the MIDAS motif), it is likely that the high affinity PA interaction with the ANTXR proteins outcompeted any potential interaction with collagen. It is not clear why PA was included in the same coimmunoprecipitations between ANTXR proteins and collagen.

So, the evidence in this study that the C5 domain does not bind ANTXR1 is very weak in my opinion, and should be recieved with caution. 